\title{
INTRODUCTORY TUTORIAL: AGENT-BASED MODELING AND SIMULATION
}

\author{
Charles M. Macal \\ Michael J. North \\ Argonne National Laboratory \\ Center for Complex Adaptive Systems Simulation $\left(\mathrm{CAS}^{2}\right)$ \\ Decision \& Information Sciences Division \\ Argonne, IL 60439 USA
}

\begin{abstract}
Agent-based simulation (ABS) is an approach to modeling systems comprised of individual, autonomous, interacting "agents." Agent-based modeling offers ways to more easily model individual behaviors and how behaviors affect others in ways that have not been available before. There is much interest in developing agent-based models for many application problem domains. Applications range from modeling agent behavior in supply chains and the stock market, to predicting the success of marketing campaigns and the spread of epidemics, to projecting the future needs of the healthcare system. Progress in the area suggests that ABS promises to have far-reaching effects on the way that businesses use computers to support decision-making and researchers use agent-based models as electronic laboratories to aid in discovery. This brief tutorial introduces agent-based modeling and simulation by describing the basic ideas of ABS, discussing some applications, and addressing methods for developing agent-based models.
\end{abstract}

\section{INTRODUCTION}

Agent-based simulation (ABS) is a simulation approach made possible by recent advances in computational modeling and software. ABS has gained increasing attention over the past several years as evidenced by the increasing numbers of articles appearing in modeling and applications journals, funded programs that call for agent-based models incorporating elements of human and social behavior, the demand for ABS courses and instructional programs, and the growing number of conferences on or that have tracks dedicated to agent-based modeling, such as the Winter Simulation Conference (WSC). Interest in ABS has steadily grown at the WSC since the first ABS tutorial we presented (Macal and North 2005).

ABS is a computational framework for simulating dynamic processes that involve autonomous agents. An autonomous agent acts on its own without external direction in response to situations the agent encounters during the simulation. Modeling a population of autonomous and heterogeneous agents that extensively interact is a defining feature of an ABS. Agent-based simulation is most commonly used to model individual decision-making and social and organizational behavior (Bonabeau 2001). The notions of behavior, decision-making, and interaction apply to modeling many kinds of system. An agent is a general concept having broad applicability. Agents often represent people, or groups of people. Agent relationships represent processes of social interaction (Gilbert and Troitzsch 1999). For example, an individual's daily activities are explicitly modeled in an ABS of infectious disease transmission to understand infection patterns arising from contact with other individuals. In a supply chain ABS, agents are firms with decision-making behaviors about material sourcing and ordering, stocking, and shipping. In an ABS composed of artificial agents, collaborating robots search the landscape and communicate their findings to collectively accomplish a task. The development of agent-based modeling tools, the availability of micro- 


\section{Macal and North}

data on agent transactions and interactions, and advances in computation have made possible a growing number of ABS applications across a variety of domains and disciplines.

Agent-based modeling is being applied to many areas, spanning human social, behavioral, cultural, physical and biological systems. Applications range from modeling ancient civilizations that have been gone for hundreds of years, to designing new markets for products that do not exist right now. Heath, Ciarallo, and Hill (2009) provide a review of agent-based modeling applications. Selected applications are listed in Table 1. All of the cited publications make the case for agent-based modeling as the preferred modeling approach for the problems addressed. The authors argue that only agent-based models can explicitly incorporate the complexity arising from individual behaviors and interactions that exist in the real world.

This tutorial provides an introduction to agent-based modeling and simulation. The goals are to show that ABS is: Useful: Why ABS is an appropriate modeling approach for a large class of problems and has advantages over conventional modeling approaches in many cases, Usable: How ABS is advancing to the point of producing portable, extensible, and transferable software, with better integrated development environments, and Used: How good ABS applications are being developed to solve practical problems.

Agent-based modeling began as the computational arm of the field of "artificial life," which is concerned with the emergence of order in nature, with the development of Swarm, the first agent-based modeling toolkit some 20 years ago (Macal 2009). Previous to that, the field of cellular automata gave the form and time and state advance mechanisms to many of the original agent-based simulations. Although ABS originated and developed quite independently of traditional Monte Carlo and discrete event simulation (DES), we attempt to present an integrated view of these disparate developmental paths in this tutorial. We refer the reader to previous papers on other introductory topics in ABS not covered here, such as the history of $\mathrm{ABS}$ and the relationships of ABS to other modeling and simulation techniques (Macal, North and Samuelson 2013; Macal and North 2010; North and Macal 2007).

This tutorial is organized as follows. The first part is on how to think about ABS (Section 2). The second part is on how to do ABS. Section 3 is a practical guide on how to do ABS, and Section 4 is on how to get started in ABS. Section 5 considers when it is appropriate to use ABS.

\section{HOW TO THINK ABOUT AGENT-BASED MODELING}

\subsection{Structure of an agent-based model}

A typical agent-based model has three elements:

- Agents, their attributes and behaviors.

- Agent relationships and methods of interaction. An underlying topology of connectedness defines how and with whom agents interact.

- Agents' environment. Agents live in and interact with their environment, in addition to other agents.

A model developer must identify, model, and program these elements to create an agent-based model. The structure of a typical agent-based model is shown in Figure 1. A computational engine for simulating agent behaviors and agent interactions is then needed to make the model run. An agent-based modeling toolkit, programming language or other implementation provides this capability. To run an agent-based model is to have agents repeatedly execute their behaviors and interactions. This process is often but not necessarily modeled to operate over a timeline, as in time-stepped, activity-based, or discrete-event simulation. 


\section{Macal and North}

Table 1: A sample of recent agent-based applications

\begin{tabular}{|c|c|}
\hline Application Area: & Agent-based Model Focus: \\
\hline Agriculture & $\begin{array}{l}\text { A spatial individual-based model prototype for assessing potential pesticide exposure of farm- } \\
\text { workers conducting small-scale agricultural production (Leyk et al. 2009) }\end{array}$ \\
\hline Air Traffic Control & $\begin{array}{l}\text { Air traffic control to analyze control policies and performance of an air traffic management facili- } \\
\text { ty (Conway 2006) }\end{array}$ \\
\hline Anthropology & $\begin{array}{l}\text { Prehistoric settlement patterns and political consolidation in the Lake Titicaca basin of Peru and } \\
\text { Bolivia (Griffin and Stanish 2007) }\end{array}$ \\
\hline Biomedical Research & $\begin{array}{l}\text { The Basic Immune Simulator, to study the interactions between innate and adaptive immunity } \\
\text { (Folcik et al. 2007) }\end{array}$ \\
\hline Crime Analysis & A realistic virtual urban environment, populated with virtual burglar agents (Malleson 2010) \\
\hline Ecology & $\begin{array}{l}\text { Investigate the trade-off between road avoidance and salt pool spatial memory in the movement } \\
\text { behavior of moose (Grosman et al. 2011) } \\
\text { Predator-prey relationships between transient killer whales and other marine mammals (Mock and } \\
\text { Testa 2007) }\end{array}$ \\
\hline Energy Analysis & $\begin{array}{l}\text { A building occupant network energy consumption decision-making model (Chen et al. 2011) } \\
\text { Application for the Smart Grid (Jackson 2010) } \\
\text { Energy investment decision making (Tobias 2008) } \\
\text { Oil refinery supply chain (Van Dam et al. 2008) }\end{array}$ \\
\hline Epidemiology & $\begin{array}{l}\text { Pandemic disease model accounting for individual behavior and demographics (Aleman et al. } \\
\text { 2010) } \\
\text { Global-scale agent model of disease transmission (Parker and Epstein 2011) }\end{array}$ \\
\hline Evacuation & $\begin{array}{l}\text { Tsunami evacuation using a modified form of Helbing's social-force model applied to agents } \\
\text { (Puckett 2009) }\end{array}$ \\
\hline Market Analysis & $\begin{array}{l}\text { Consumer marketing model developed in collaboration with a Fortune } 50 \text { firm (North et al. 2009) } \\
\text { Consumer airline market share (Kuhn et al. 2010) } \\
\text { Simulation that models the possibilities for a future market in sub-orbital space tourism (Charania } \\
\text { et al. 2006) }\end{array}$ \\
\hline Social Networks & $\begin{array}{l}\text { Model of email-based social networks, in which individuals establish, maintain and allow atrophy } \\
\text { of links through contact-lists and emails (Menges et al. 2008) }\end{array}$ \\
\hline
\end{tabular}

\subsection{Agents}

There is not universal agreement on the precise definition of the term agent in the context of ABS. It is the subject of much discussion and occasional debate. The issue is more than an academic one, as it often surfaces when one makes a claim that their model is agent-based or when one is trying to discern whether such claims made by others have validity. There are important implications of the term agent-based when used to describe a model in terms of the model's capabilities or potential capabilities that could be attained through relatively minor modification. A formal definition of agent is beyond the scope of this paper; in the literature informal descriptions of agent tend to agree on more points than they disagree. Some modelers consider any type of independent component, whether it be a software component or a model to be an agent (Bonabeau 2001). Some authors insist that a component's behavior must also be adaptive in order for it to be considered an agent. Casti (1997) argues that agents should contain both base-level rules 


\section{Macal and North}

for behavior as well as a higher-level set of "rules to change the rules." The base-level rules provide responses to the environment, while the rules-to-change-the-rules provide adaptation. Jennings' (2000) computer science-based view of agent emphasizes the essential agent characteristic of autonomous behavior.

Agents Interacting in Agent Space (Grid Topology)

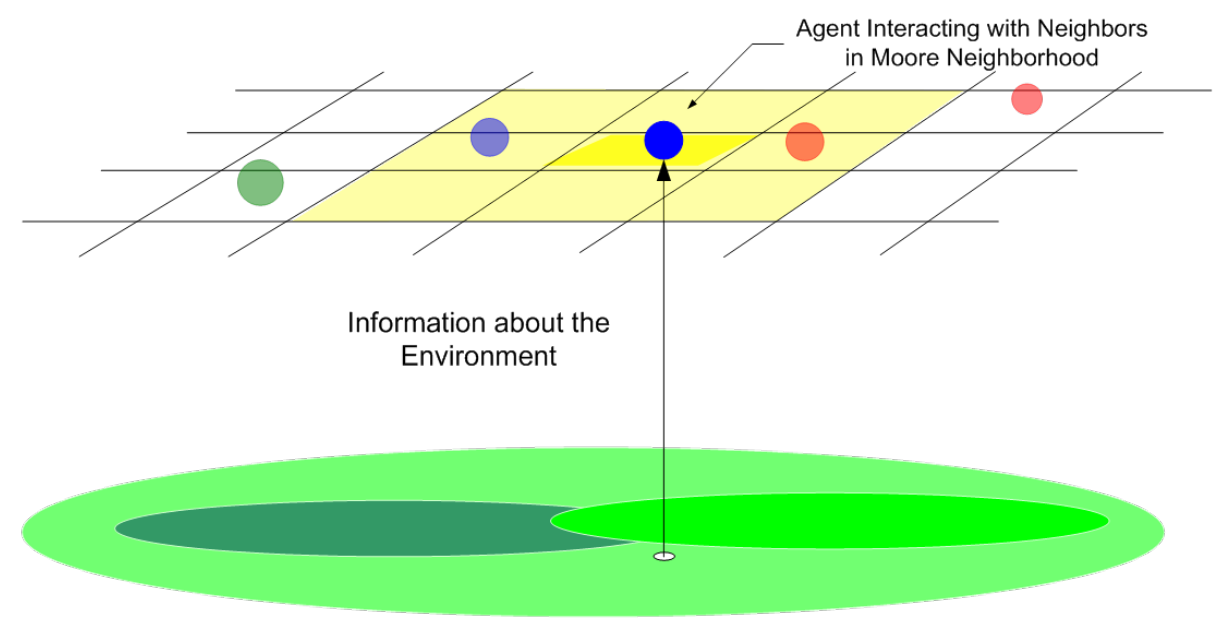

Environment

Figure 1: General elements of an agent-based model

For practical modeling purposes, we consider agents to have certain properties and attributes, as follows (Figure 2):

Modularity. Agents are modular or self-contained. An agent is an identifiable, discrete individual with a set of characteristics or attributes, behaviors, and decision-making capability. The modularity requirement implies that an agent has a boundary, and one can easily determine whether something (that is, an element of the model's state) is part of an agent, is not part of an agent, or is a characteristic shared among agents.

Autonomy. An agent is autonomous and self-directed. An agent can function independently in its environment and in its interactions with other agents, generally from a limited range of situations that are of interest and that arise in the model. When we refer to an agent's behavior, we refer to a process that links the information the agent senses from its environment and interactions to its decisions and actions.

Sociality. An agent is social, interacting with other agents. Common agent interaction protocols include contention for space and collision avoidance, agent recognition, communication and information exchange, influence, and other domain-or application-specific mechanisms.

Conditionality. An agent has a state that varies over time. Just as a system has a state consisting of the collection of its state variables, an agent also has a state that represents its condition, the essential variables associated with its current situation. An agent's state consists of a set or subset of its attributes. The state of an agent-based model is the collective states of all the agents along with the state of the environment. An agent's behaviors are conditioned on its state. As such, the richer the set of an agent's possible states, the richer the set of behaviors that an agent can have.

Agents often have additional properties, which may or may not be considered as requisite properties for agency. An agent may have explicit goals that drive its behavior, not necessarily objectives to maximize as much as criteria against which to assess the effectiveness of its decision and actions. An agent may have the ability to learn and adapt its behaviors based on its experiences. A common reason for modeling 


\section{Macal and North}

a system as an ABS is to consider agent learning and adaptation. At the individual level, learning and adaptation can be modeled as agent behaviors. Individual learning and adaptation requires an agent to have memory as a dynamically updated attribute of the agent. At the population level, adaptation can be modeled by allowing agents to enter and leave the population, with the more successful agents increasing their relative numbers in the population over time.

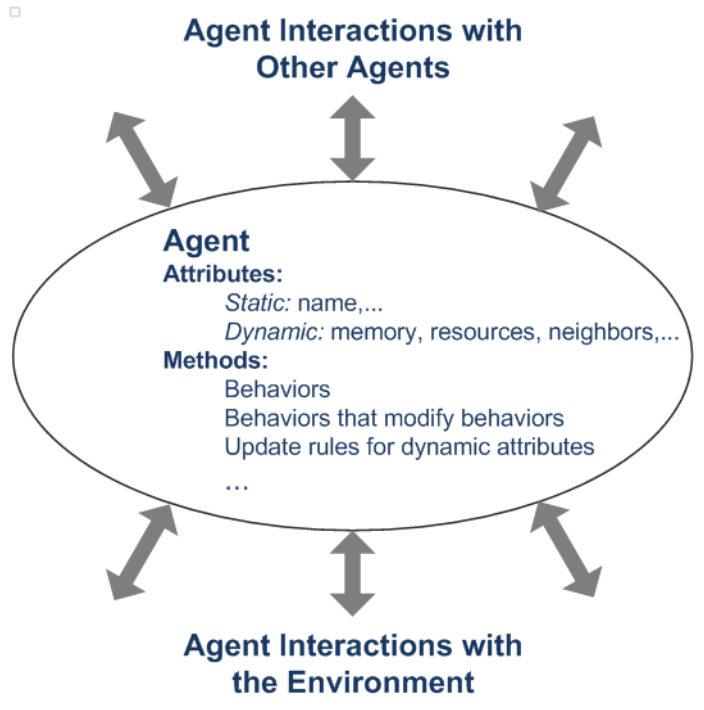

Figure 2: A typical agent

\subsection{Agent Relationships}

Agent-based modeling concerns itself with modeling agent relationships and agent interactions as much as it does modeling agents and agent behaviors. The primary issues of modeling agent interactions are specifying who is, or could be, connected to who, and the dynamics governing the mechanisms of the interactions. For example, an agent-based model of Internet growth would include mechanisms that specify who connects to who, why, and when.

Common topologies for representing social agent interactions are as follows: (1) Soup. A nonspatial model in which agents have no locational attribute, (2) Grid or lattice. Cellular automata represent agent interaction patterns and available local information by a grid or lattice; cells immediately surrounding an agent are its neighborhood. An agent's location is the grid cell index. (3) Euclidean space. Agents roam in 2D or 3D spaces. An agent's location is its relative (grid) or geospatial (lat-long) coordinates. (4) Geographic Information System (GIS). Agents move over and interact with realistic geo-spatial landscapes. An agent's location is a geographical unit (e.g., zip code) or geospatial coordinates. (5) Networks. Networks may be static (links pre-specified) or dynamic (links determined endogenously). An agent's location is relative to other nodes in the network.

No matter what agent-interaction topology is used in an agent-based model to connect the agents, the essential idea is that agents only interact at any given time with a limited number of other agents out of all the agents in the population. This notion is implemented by defining a local neighborhood and limiting interaction to a small number of agents that are located in that neighborhood. This is not to say that agents need to be located in close proximity to one another spatially to be able to interact. The network topology allows agents to be linked on the basis of relationships in addition to proximity. For example, an agent may be a member of many networks, e.g., proximity, social, familial relationship, ideological, etc. 


\section{Macal and North}

\subsection{Emergence in Agent-based Models}

One of the motivations for agent-based modeling is its ability to capture emergence. Emergence is important or interesting behavior exhibited by the agents or the process under study, which is not explicitly modeled at the outset, but which appears as a result of agent interactions when the model is run. Emergent behavior is often unexpected. How to identify unexpected behavior in simulation output is an area of further research. Even simple agent-based models that are completely described by simple, deterministic rules and based only on local information can self-organize and sustain themselves in ways that have not been explicitly programmed into the models. Emergence can be illustrated by simple agent-based models such as Life and Boids. More complex models of the kind that people are likely to build to represent realworld phenomenon can also exhibit emergent behavior resulting from agent interactions. Agent-based modeling algorithms based on emergence have led to optimization techniques, such as ant colony optimization and particle swarm optimization, that have been used to solve practical problems (Bonabeau, Dorigo, and Theraulaz 1999; Barbati, Bruno and Genovese 2011).

\section{HOW TO DO AGENT-BASED MODELING}

\subsection{Thinking Through an Agent Model}

Agent-based model development follows the general steps of developing any model with the additions of agent-related tasks (Figure 3). It is useful to ask a series of agent-specific questions before developing an agent-based model (Table 2). (It is also beneficial to continue to ask many of the questions during development, since ABS development is an iterative process of refinement.) The answers to these questions help define the scope and level of detail, granularity, appropriate to modeling the system. They imply the resources required for successfully completing the project as well as help identifying likely bottlenecks to development.

\subsection{Modeling Agent Systems}

Identifying agents, accurately specifying their behaviors, and appropriately representing agent interactions are the keys to developing useful agent models. One begins developing an agent-based model by identifying the agent types (classes) along with their attributes. Agents are generally the decision-makers in a system whether they be human, organizational, or automated. Once the agents are defined, agent behaviors are specified. One needs to have a theory of agent behavior as a basis for modeling agent behavior. For example, a normative model in which agents attempt to optimize a well-defined objective can be a useful starting point to eventually developing more descriptive and domain-specific behavioral heuristics. Alternatively, one may begin with a bounded rationality model or a generic behavioral heuristic, such as anchoring and adjustment, to describe agent behavior, or a formal behavioral modeling framework such as the Belief-Desire-Intent (BDI) model (Wooldridge 2009) may be appropriate.

In addition to agents, an agent-based model consists of agent relationships. One defines the agent relationships and then adds the methods that control which agents interact, when they interact, and how they interact. 


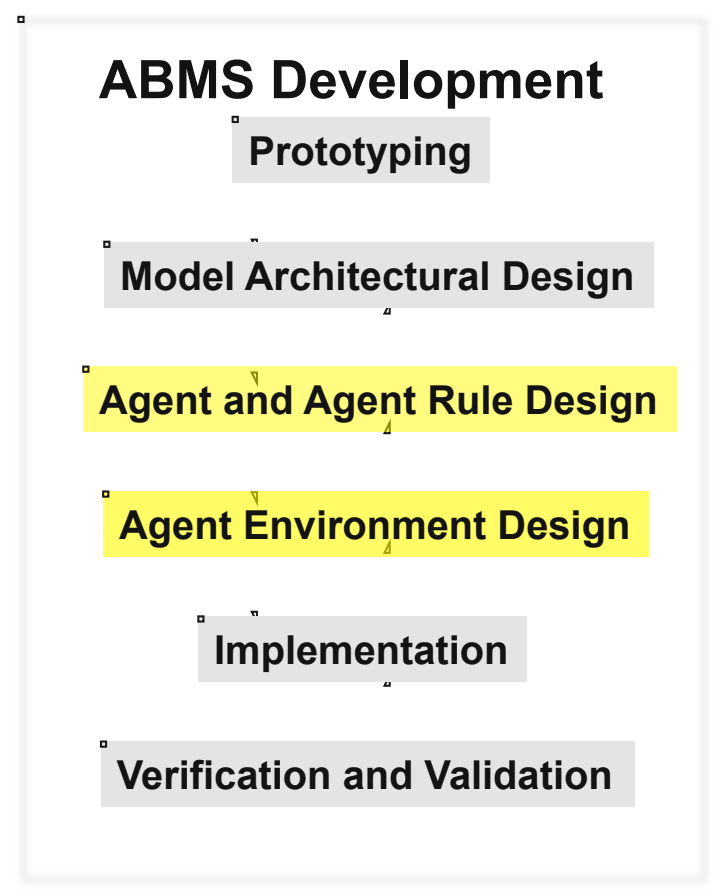

Figure 3: Agent -based model and simulation (ABMS) development process

\subsection{Designing Agent-based Models}

Design is a key phase in building agent-based models. Modern software practices are based on a template design approach in which recurring elements are codified and reused for new applications. This approach has proven very valuable in designing models as well as software. For example, the Unified Modeling Language (UML) provides a set of tools in the form of diagrams for software system design and representation that is independent of computer code implementation (Booch, Jacobson and Rumbaugh 2005). Several formats have been proposed for describing agent-based model designs. Chief among these standards is Grimm et al.'s (2006) Overview, Design Concepts, and Details (ODD) protocol. ODD describes models using a three-part approach: overview, concepts, and details. The model overview includes a statement of the model's intent, a description of the main variables, and a discussion of the agent activities and timing. The design concepts include a discussion of the foundations of the model, and the details include the initial setup configuration, input value definitions, and descriptions of any embedded models (Grimm et al. 2006). North and Macal (2013) discuss helpful process and product design patterns for agent-based modeling. An agent-based modeler refers to existing design patterns to model important features of a real-world system, such as, for example, how time should be treated in a model.

\subsection{Advanced Agent-based Modeling}

Often, an agent-based modeler would like to include a variety of advanced capabilities in their model. These capabilities include distributed computing implementations, artificial intelligence and machine learning algorithms, geographical information systems (GIS), connections to relational databases, version control systems (especially if there are multiple developers working on a project), and integrated development environments (IDEs). It is often useful to first develop a core model that includes these capabilities as connections or "stubs" to ensure the core model design is acceptable and to verify that scaling up 


\section{Macal and North}

the design appears feasible. Agent-based modeling and software toolkits usually provide advanced capabilities such as these.

Table 2: Questions to Ask Before Developing an Agent-based Model

\begin{tabular}{l} 
Model Purpose and Value-added of Agent-based Modeling: \\
What specific problem is the model being developed to address? \\
What specific questions should the model answer? \\
What kind of information should the model provide to help make or support a decision? \\
Why might agent-based modeling be a desirable approach? \\
What value-added does agent-based modeling bring to the problem that other modeling approaches cannot bring? \\
All About Agents: \\
What should the agents be in the model? \\
Who are the decision makers in the system? \\
What are the entities that have behaviors? \\
Where might the data come from, especially on agent behaviors, for such a model? \\
Agent Data: \\
What data on agents is simply descriptive (static attributes)? \\
What agent attributes are calculated endogenously by the model and updated for the agents (dynamic attributes)? \\
What is the agents' environment? How do the agents interact with the environment? Is agent mobility through \\
space an important consideration? \\
Agent Behaviors: \\
What agent behaviors are of interest? \\
What decisions do the agents make and what information is required to make such decisions? \\
What behaviors are being acted upon? \\
What actions are being taken by the agents? \\
How would we represent the agent behaviors? By If-Then rules? By adaptive probabilities, such as in reinforce- \\
ment learning? By regression models or neural networks? \\
\hline Agent Interactions: \\
How do the agents interact with each other? \\
How do the agents interact with the environment? \\
How expansive or focused are agent interactions? \\
Agent Recap: \\
How do we design a set of experiments to explore the importance of uncertain behaviors, data and parameters? \\
How might we validate the model, especially the agent behaviors and the agent interaction mechanisms? \\
\hline
\end{tabular}

\subsection{ABS Software and Toolkits}

Agent-based modeling can be done using general, all-purpose software or programming languages, or can be done using specially designed software and toolkits that address the specific requirements for modeling agents. Agent modeling can be done in the small, on the desktop, or in the large, using large-scale computing clusters, or it can be done at any scale in-between. Projects often begin small, using one of the desktop ABS tools, or whatever tool or programming language the developers are familiar with. The initial prototype then grows in stages into a larger-scale agent-based model, often using dedicated ABS toolkits. Often one begins developing their first agent model using the approach that one is most familiar with, or the approach that one finds easiest to learn given their background and experience.

We distinguish several approaches to building ABS applications in terms of the scale of the software that one can apply according to the following continuum: 
Desktop Computing for ABS Application Development:

- Spreadsheets: Excel using the macro programming language VBA

- Dedicated Agent-based Prototyping Environments: Repast Simphony, NetLogo

- General Computational Mathematics Systems: MATLAB, Mathematica

Large-Scale (Scalable) Agent Development Environments:

- Repast

- Swarm

- MASON

- AnyLogic

General (Object-Oriented) Programming Languages:

- $\mathrm{C}++$

- Java

- Python

Desktop ABS can be used to learn agent modeling, prototype basic agent behaviors, and perform limited analyses. Desktop agent-based models can be simple, designed and developed independently in a period of a few days by a single computer-literate modeler using tools learned in a few days or weeks. Desktop agent modeling can be used to explore the potential of ABS with relatively minor time and training investments, especially if one is already familiar with the tool.

Spreadsheets, such as Microsoft Excel, are in many ways the simplest approach to modeling. It is easier to develop models with spreadsheets than with many of the other tools, but the resulting models generally allow limited agent diversity, restrict agent behaviors, and have poor scalability compared to the other approaches designed specifically for agent modeling. Agent-based modeling in spreadsheets requires some macro-programming in a language such as VBA (Visual Basic for Applications), the macro programming language for Excel and other Microsoft Office applications. Significant agent models have been developed entirely using spreadsheets. In a previous WSC paper, we described the detailed spreadsheet implementation of a simple, spatial agent-based "shopper model" (Macal and North 2007).

General-purpose desktop computational mathematics systems (CMS) with integrated development environments (IDEs), such as MATLAB and Mathematica, can be used to develop agent models, although the agent-specific functionality has to be written by the developer from scratch, as there are no dedicated libraries or modules that focus on agent-based modeling. The basic requirement is knowledge of how to program in a scripting language. CMS environments have rich mathematical functions, and nearly any mathematical relation or function that can be numerically calculated is available within these tools or their add-on libraries. In some cases, the tools even support symbolic processing and manipulation, which is useful for systems of equations that can be solved analytically and can be exploited quite effectively to do agent-based modeling. If a CMS environment is already familiar to a developer, this can be a good place to start agent-based modeling (Macal 2004).

Special-purpose agent tools, such as NetLogo, provide facilities for agent modeling. NetLogo is a free ABS environment developed at Northwestern University's Center for Connected Learning and ComputerBased Modeling (Wilensky 2013). The most directly visible common trait shared by the various prototyping environments is that they are designed to get first-time users started as quickly as possible. The NetLogo language uses a modified version of the Logo programming language (Harvey 1997). NetLogo was originally developed to support ABS education at all levels, but it can be used to develop a wide range of applications. NetLogo provides a graphical environment to create programs that control graphic "turtles" that reside in a world of "patches" that is monitored by an "observer." Agents may also interact 


\section{Macal and North}

over networks. NetLogo continues as a subject of active development and new versions with expanded capabilities are released periodically.

Many large-scale ABS software environments are now freely available. These include Repast (North, Collier and Vos, 2006; North et al. 2013), Swarm (Minar et al. 1996), NetLogo (Wilensky 2013) and MASON (GMU 2013) among many others. Proprietary toolkits are also available such as AnyLogic (XJ Technologies 2013). Tobias and Hoffman (2004) and Nikolai and Madey (2009) review Java-based agent modeling toolkits.

Swarm was the first ABS software development environment, launched in 1994 at the Santa Fe Institute. Swarm was originally written in Objective C and was later fitted with a Java interface. Following the original Swarm innovation, the Repast (REcursive Porous Agent Simulation Toolkit) toolkit was developed as a pure Java implementation (North, Collier, and Vos 2006). Repast Simphony (Repast S) is the latest version of Repast, designed to provide visual point-and-click tools for agent model design, agent behavior specification, model execution, and results examination. The Repast $\mathrm{S}$ runtime environment includes automated results analysis and connections to a variety of spreadsheet, visualization, data mining, and statistical analysis tools, virtually all of which are free and open source. Repast Simphony 2.0 also includes ReLogo, a new Logo-like interface for specifying agent models (Ozik 2013).

As computational capabilities continue to advance in both hardware and software, new capabilities are continuously being incorporated into the latest versions of ABS toolkits. The field is advancing rapidly toward highly scalable, high productivity agent development environments that are easy to learn and use.

\section{HOW TO GET STARTED WITH AGENT-BASED MODELING}

Many people ask how to get started with agent-based modeling. A good background in simulation modeling in the traditional fields of discrete-event simulation, Monte Carlo simulation, or even system dynamics is an excellent prerequisite but is not essential, as the field of agent-based modeling was not originally founded on these fields or the software that supports them. Some universities offer courses on agentbased modeling, but not very many at this time. There is not even a generally agreed upon agent-based modeling curriculum for the modeling and simulation community, but Macal and North (2013) attempt to get that discussion rolling through their experiences with workshops and tutorials such as this one. Many people have become successful agent-based modelers, including the authors, by independently following a path such as this:

1. Read about introductory agent-based modeling,

2. Review some good, simple applications in the literature,

3. Download, play with, and inspect some available pre-built ABS demonstration models,

4. Attend conferences devoted to agent-based modeling or that have significant focus on ABS,

5. Define a problem meaningful to you to address with agent-based modeling (see Table 2), and

6. Develop some simple agent-based models (prototypes) in available ABS toolkits.

Having gone through these steps allows one to start thinking about how to develop larger-scale and serious agent-based models. The most important point to make is that there is no substitute for learning about agent-based modeling than to get one's hands dirty and actually build an agent-based model. This is true even if the ultimate goal is not to become a full-time agent-based modeler. Steps 5 and 6 were discussed in the previous section. Steps 1-4 are discussed below.

A single comprehensive source for reading all about ABM does not exist. Good introductions to ABM include the web sites by Axelrod and Tesfatsion (2013), the ACE web site also by Tesfatsion (2013), and the new web site by Railsback and Grimm (2013). The book by Epstein and Axtell (1996) is often regarded as launching the field of social agent simulation in a sustained way. It includes simple, but 


\section{Macal and North}

elegant, models of various social processes that are still being elaborated upon. The early paper by Bonabeau (2001) remains as one of the most cited and readable papers on the motivations for ABS. The book by Gilbert and Troitzsch (2005) is widely read and provides a highly readable overview of the field including how to construct simple ABS. Our book (North and Macal 2007) is designed to provide a broad non-technical introduction to ABS in terms of how to think about and do ABS as well as providing terminology and language for becoming conversant in agent-based modeling.

Good ABM applications are scattered throughout the literature across many disciplines. There is no single publication source for ABM applications, but the online Journal of Artificial Societies and Social Simulation (JASSS) has provided a consistent outlet for agent-based models for many years (http://jasss.soc.surrey.ac.uk/JASSS.html).

Disciplines often produce their own overview publications on agent-based modeling specific to their discipline. These can serve as valuable resources for understanding the value of using ABM in a discipline and include key references for the domain. For example, disciplinary ABM overview and survey papers include: marketing (Rand and Rust 2011), economics (Cristelli, Pietonero and Zaccaria 2011), financial economics (LeBaron 2005), transportation (Bernhardt 2013), electric power markets (Weidlich and Veit 2008), geographical information systems (GIS) (Brown et al. 2005), and many other areas. Simple Google searches on "agent based model" or multi agent system model" yield many application papers.

It can be very useful to visit the web sites for the ABM toolkits and download the software (NetLogo or Repast, for example) or trial versions (AnyLogic, for example). Demonstration examples are provided that give a good idea of how agent-based models are constructed and of the software's capabilities.

Several conferences have a focus on agent-based modeling or tracks devoted to ABS. The annual Winter Simulation Conference tends to have a full track of proceedings papers devoted to agent-based simulation and applications. Our estimates are that about $20 \%$ of the papers in WSC are on agent-based simulation. The annual MABS (Multi-Agent-Based Simulation) workshop, which is part of IAAMAS (International Conference on Autonomous Agents and Multi-Agent Systems), focuses on agent-based modeling "from the standpoint of the multiagent systems community of engineering and the social/economic/organizational sciences" (https:/sites.google.com/site/mabsworkshop/). The annual Computational Social Science Society of the Americas (CSSSA) conference, formerly NAACSOS, focuses on "Computational Social Science (CSS), a scientific discipline where computational methods and simulation models of social dynamics are employed to offer new insights into social phenomena beyond what is available with traditional social science methods" (http://computationalsocialscience.org/csssa2013). Other conferences such as the annual INFORMS meeting (https://www.informs.org/) and the annual MORSS (Military Operations Research Society Symposium, http://www.mors.org/) often have significant numbers of presentations involving agent-based models, and these number have steadily grown over the past 10 years.

\section{WHY AND WHEN ABS}

We conclude by offering some ideas on the situations for which agent-based modeling can offer distinct advantages to conventional simulation approaches such as discrete event simulation (Law 2007), system dynamics (Sterman 2000) and other quantitative modeling techniques. Axtell (2000) discusses several reasons for agent-based modeling especially compared to traditional approaches to modeling economic systems. When is it beneficial to think in terms of agents? When one or more of the following criteria are satisfied:

- When the problem has a natural representation as being comprised of agents

- When there are decisions and behaviors that can be well-defined

- When it is important that agents have behaviors that reflect how individuals actually behave (if known)

- When it is important that agents adapt and change their behaviors 


\section{Macal and North}

- When it is important that agents learn and engage in dynamic strategic interactions

- When it is important that agents have dynamic relationships with other agents, and agent relationships form, change, and decay

- When it is important to model the processes by which agents form organizations, and adaptation and learning are important at the organization level

- When it is important that agents have a spatial component to their behaviors and interactions

- When the past is no predictor of the future because the processes of growth and change are dynamic

- When scaling-up to arbitrary levels is important in terms of the number of agents, agent interactions and agent states

- When process structural change needs to be an endogenous result of the model, rather than an input to the model

- When the process might behave in important possibly unsuspected ways, which running the model would display as emergent behavior

\section{ACKNOWLEDGMENTS}

This work was supported by the U.S. Department of Energy under contract number DE-AC0206CH11357. Portions of this tutorial have appeared in previous tutorial papers presented at the Winter Simulation Conference for 2005 - 2009.

\section{REFERENCES}

Aleman, D., T. Wibisono, and B. Schwartz. 2009. "Accounting for Individual Behavior and Demographics in Pandemic Disease Modeling." Proc. 2009 Winter Simulation Conf., M. D. Rossetti, R. R. Hill, B. Johansson, A. Dunkin and R. G. Ingalls, eds., pp. 86-98, Wiley-IEEE Press.

Axelrod, R., and L. Tesfatsion. 2013. "On-Line Guide for Newcomers to Agent-Based Modeling in the Social Sciences," www.econ.iastate.edu/tesfatsi/abmread.htm [Accessed June 30, 2013].

Axtell, R. 2000. Why Agents? On The Varied Motivations for Agent Computing in the Social Sciences, Working Paper 17, Center on Social and Economic Dynamics, Brookings Institution, Washington, D.C.

Barbati, M., G. Bruno, and A. Genovese. 2011. "Applications of agent-based models for optimization problems: a literature review," Expert Systems with Applications, doi: 10.1016/j.eswa. 2011.12.015.

Bernhardt, K. L. S. 2013. "Agent-Based Modeling in Transportation." 70 Transportation Research Circular E-C113: Artificial Intelligence in Transportation, pp. 72-80.

Bonabeau, E. 2001. "Agent-Based Modeling: Methods and Techniques for Simulating Human Systems." In Proc. National Academy of Sciences 99(3): 7280-7287.

Bonabeau, E., M. Dorigo, and G. Theraulaz. 1999. Swarm Intelligence: From Natural To Artificial Systems. Oxford: Oxford University Press.

Booch, G., I. Jacobson, and J. Rumbaugh. 2005. The Unified Modeling Language User Guide, $2^{\text {nd }}$ ed. Addison-Wesley.

Brown, D. G., R. Riolo, D. T. Robinson, M. North, and W. Rand. 2005. "Spatial processes and data models: Toward integration of agent-based models and GIS." Journal of Geographical Systems 7(1): 2547.

Casti, J. 1997. Would-Be Worlds: How Simulation Is Changing the World of Science. New York: Wiley.

Charania, A. C., J. R. Olds and D. DePasquale. 2006. Sub-Orbital Space Tourism Market: Predictions of the Future Marketplace Using Agent-Based Modeling. SpaceWorks Engineering, Inc., Atlanta, GA.

Chen, J., J. E. Taylor, H.-H. Wei. 2011. "Modeling Building Occupant Network Energy Consumption Decision-making: The Interplay Between Network Structure and Conservation." Energy and Buildings Available online 26 December. 


\section{Macal and North}

Conway, S. R. 2006. "An Agent-Based Model for Analyzing Control Policies and the Dynamic ServiceTime Performance of a Capacity-Constrained Air Traffic Management Facility." In Proceedings of ICAS 2006 - 25th Congress of the International Council of the Aeronautical Sciences. Hamburg, Germany, 3-8 Sep. 2006.

Cristelli, M., L. Pietonero, and A. Zaccaria. 2011. Critical Overview of Agent-Based Models for Economics. http://arxiv.org/pdf/1101.1847.pdf [Accessed July 1, 2013].

Epstein, J. M., and R. Axtell. 1996. Growing Artificial Societies: Social Science From The Bottom Up. Cambridge, MA: MIT Press.

Folcik, V. A., G. C. An, and C. G. Orosz. 2007. "The Basic Immune Simulator: An Agent-Based Model to Study the Interactions between Innate and Adaptive Immunity." Theoretical Biology and Medical Modelling, 4(39).

Gilbert, N., and K. Troitzsch. 2005. Simulation for the Social Scientist. McGraw-Hill. 2nd ed.

GMU (George Mason University). 2009. MASON Home Page. http://cs.gmu.edu/ eclab/projects/mason/ [Accessed July 1, 2013].

Griffin, A. F., and C. Stanish. 2007. "An Agent-Based Model of Prehistoric Settlement Patterns and Political Consolidation in the Lake Titicaca Basin of Peru and Bolivia, Structure and Dynamics." eJournal of Anthropological and Related http://repositories.cdlib.org/imbs/socdyn/sdeas/vol2/iss2/art2. [Accessed July 1, 2013].

Grimm, V., U. Berger, F. Bastiansen, S. Eliassen, V. Ginot, J. Giske, J. Goss-Custard, T. Grand, S. K. Heinz, G. Huse, A. Huth, J. U. Jepsen, C. Jørgensen, W. M. Mooij, B. Müller, G. Pe'er, C. Piou, S. F. Railsback, A. M. Robbins, M. M. Robbins, E. Rossmanith, N. Rüger, E. Strand, S. Souissi, R. A. Stillman, R. Vabø, U. Visser, and D. L. DeAngelis. 2006. "A Standard Protocol for Describing Individual-Based and Agent-Based Models.” Ecological Modelling 198(1-2):115-126.

Grosman, P. D., J. A. G. Jaeger, P. M. Biron, C. Dussault, and J.-P. Ouellet. 2011. "Trade-off Between Road Avoidance and Attraction by Roadside Salt Pools in Moose: An Agent-Based Model to Assess Measures for Reducing Moose-Vehicle Collisions.” Ecological Modelling 222(8):1423-1435.

Harvey, B. 1997. Computer Science Logo Style. Boston: MIT Press.

Heath, B. L., F. Ciarallo, and R. R. Hill. 2009. "A Survey Of Agent-Based Modeling Practices (January 1998 to July 2008)." Journal of Artificial Societies and Social Simulation 12(4) October.

Jackson, J. 2010 "Improving energy efficiency and smart grid program analysis with agent-based end-use forecasting models." Energy Policy doi:10.1016/j.enpol.2010.02.055.

Jennings, N. R. 2000. "On Agent-Based Software Engineering." Artificial Intelligence 117:277-296.

Kuhn Jr, J. R., J. F. Courtney, B. Morris, and E. R. Tatara. 2010. "Agent-Based Analysis and Simulation of the Consumer Airline Market Share for Frontier Airlines." Knowledge-Based Systems 23(8): 875882.

Law, A. M. 2007. Simulation Modeling and Analysis. 4th ed. New York: McGraw-Hill.

LeBaron, B. 2005. Agent-based Computational Finance. http://people.brandeis.edu/ blebaron/wps/hbook.pdf [Accessed June 30, 2013].

Leyk, S., C. R. Binder, and J. R. Nuckols. 2009. "Spatial Modeling of Personalized Exposure Dynamics: The Case of Pesticide Use in Small-Scale Agricultural Production Landscapes of the Developing World." International Journal of Health Geographics, 8:17. doi:10.1186/1476-072X-8-17.

Macal, C. M. 2004. "Agent-Based Modeling and Social Simulation with Mathematica and MATLAB." in Proc. Agent 2004 Conference on Social Dynamics: Interaction, Reflexivity and Emergence, edited by C. Macal, C., D. Sallach, and M. North, Chicago, IL, Oct. 7-9 http://www.dis.anl.gov/agent20XY/proceedings/Agent2004.pdf, pp. 185-204 [Accessed July 1, 2013].

Macal, C. M. 2009. "Agent Based Modeling and Artificial Life." in Encyclopedia of Complexity and Systems Science, edited by R. Meyers, 112-131. Springer. ISBN 978-0-387-75888-6. 
Macal, C., and M. North. 2005. "Tutorial on Agent-based Modeling and Simulation" Proc. 2005 Winter Simulation Conference, M. E. Kuhl, N. M. Steiger, F. B. Armstrong, and J. A. Joines, eds., Orlando, FL, Dec. 4-7, pp. 2-15, available at http://www.informs-sim.org/wsc05papers/002.pdf [Accessed July $1,2013]$.

Macal, C. M., and M. J. North. 2007. “Agent-based Modeling and Simulation: Desktop ABMS.” In Proceedings of the 2007 Winter Simulation Conference, edited by S. G. Henderson, B. Biller, M.-H. Hsieh, J. Shortle, J. D. Tew, and R. R. Barton, 95-106. Available at http://www.informssim.org/wsc07papers/011.pdf [Accessed July 1, 2013] .

Macal C. M. and M. J. North. 2010. "Tutorial on Agent-Based Modeling and Simulation." Journal of Simulation 4:151-162. New York, NY USA: Palgrave Macmillan.

Macal, C. M., and M. J. North. 2013. "Successful approaches for teaching agent-based simulation." Journal of Simulation 7(1): 1-11, February.

Macal, C. M., M. J. North and D.A. Samuelson. 2013. “Agent-based Simulation.” Saul I. Gass and Micahel Fu (eds.), Encyclopedia of Operations Research and Management Science, $3^{\text {rd }}$ ed., http://www.springer.com/business+\%26+management/operations+research/book/978-1-4419-1154-4, (in press).

Malleson, N. 2010. “Agent-Based Modelling of Burglary.” PhD Thesis, School of Geography, University of Leeds. Accessed August 2011.

http://www.geog.leeds.ac.uk/fileadmin/downloads/school/people/postgrads/n.malleson/thesisfinal.pdf.

Menges, F., B. Mishra, and G. Narzisi. 2008. "Modeling And Simulation of E-Mail Social Networks: A New Stochastic Agent-Based Approach." In Proceedings of the 2008 Winter Simulation Conference, edited by S. J. Mason, R. R. Hill, L. Mönch, O. Rose, T. Jefferson, and J. W. Fowler, 2792-2800. Piscataway, New Jersey: Institute of Electrical and Electronics Engineers, Inc. https://collaboration.vbi.vt.edu/download/attachments/25953407/menges08.modeling-and-simulationof-email-social-networks.pdf [Accessed July 1, 2013].

Minar, N., R. Burkhart, C. Langton, and M. Askenazi. 1996. "The Swarm Simulation System, A Toolkit for Building Multi-Agent Simulations." Working Paper 96-06-042, Santa Fe Institute, Santa Fe, NM.

Mock, K. J., and J. W. Testa, 2007. An Agent-Based Model of Predator-Prey Relationships between Transient Killer Whales and Other Marine Mammals. University of Alaska Anchorage, Anchorage, AK, May 31, 2007. www.math.uaa.alaska.edu/ orca/ [Accessed July 1, 2013].

Nikolai, C., and G. Madey. 2009. "Tools of the Trade: A Survey of Various Agent Based Modeling Platforms." Journal of Artificial Societies and Social Simulation 12(2). http://jasss.soc.surrey.ac.uk/12/2/2.html [Accessed July 1, 2013].

North, M. J., and C. M. Macal. 2007. Managing Business Complexity: Discovering Strategic Solutions with Agent-Based Modeling and Simulation. Oxford: Oxford University Press.

North, M.J. and C. M. Macal. 2013. Product and process patterns for agent-based modelling and simulation. Journal of Simulation. advance online publication, 12 April 2013; doi:10.1057/jos.2013.4.

North, M., N. Collier, and J. Vos. 2006. "Experiences in Creating Three Implementations of the Repast Agent Modeling Toolkit." ACM Transactions on Modeling and Computer Simulation 16(1):1-25.

North, M. J., N. T. Collier, J. Ozik, E. R. Tatara, C. M. Macal, M. J. Bragen, and P. J. Sydelko. 2013. "Complex adaptive systems modeling with Repast Simphony" Complex Adaptive Systems Modeling 1:3. http://www.casmodeling.com/content/1/1/3.

North, M., C. Macal, J. St. Aubin, P. Thimmapuram, M. Bragen, J. Hahn, J. Karr, N. Brigham, M. Lacy, and D. Hampton. 2009. "Multi-scale Agent-based Consumer Market Modeling." Complexity 15(5): 37-47 (May/June).

Ozik, J., N. T. Collier, J. T. Murphy, and M. J. North. 2013. "The ReLogo Agent-based Modeling Language." In Proceedings of the 2013 Winter Simulation Conference, edited by R. Pasupathy, S.-H. Kim, A. Tolk, R. Hill, and M. E. Kuhl. 


\section{Macal and North}

Parker J., and J. Epstein. 2011. "A Distributed Platform for Global-scale Agent-based Models of Disease Transmission.” ACM Trans. Model. Comput. Simul. 22(1) Article 2.

Puckett, R. R. 2009. "Multi-Agent Crowd Behavior Simulation for Tsunami Evacuation." Masters Thesis, Department of Information and Computer Sciences, University of Hawaii, May. Accessed August 12, 2011. http://www2.hawaii.edu/ puckett/research/past/tsunami.pdf.

Railsback, S. F. and V. Grimm. 2013. "Agent-based and Individual-based Modeling: A Practical Introduction." http://www.railsback-grimm-abm-book.com/ [Accessed June 30, 2013].

Rand, W. M., and Rust, R. T. 2011. "Agent-Based Modeling in Marketing: Guidelines for Rigor." International Journal of Research in Marketing 28(3): 181-193.

Sterman, J. D. 2000. Business Dynamics: Systems Thinking and Modeling for a Complex World. Boston: Irwin McGraw-Hill.

Tesfatsion, L. 2013. "ACE web site: Agent-Based Computational Economics: Growing Economies From The Bottom Up." www.econ.iastate.edu/tesfatsi/ace.htm. [Accessed June 30, 2013].

Tobias, R., and C. Hofmann. 2004. "Evaluation of Free Java-Libraries for Social-Scientific Agent Based Simulation." Journal of Artificial Societies and Social Simulation 7(1), Jan. 31.

Van Dam, K. H., A. Adhitya, R. Srinivasan and Z. Lukszo. 2008. "Benchmarking numerical and agentbased models of an oil refinery supply chain." Computer-Aided Chemical Engineering 25.

Weidlich, A., and D. Veit. 2008. "A critical survey of agent-based wholesale electricity market models." Energy Economics 30(4): 1728-1759, http://dx.doi.org/10.1016/j.eneco.2008.01.003.

Wilensky, U. 2013. Netlogo, Center for Connected Learning and Computer-Based Modeling, Northwestern University: Evanston, IL USA. http://ccl.northwestern.edu/netlogo/. [Accessed July 1, 2013].

Wittmann, T. 2008. Agent-based models of energy investment decisions. Heidelberg: Physica-Verlag. Wooldridge, M. 2009. An Introduction to MultiAgent Systems. $2^{\text {nd }}$ ed. John Wiley.

XJ Technologies. 2013. AnyLogic Home Page. http://www.xjtek.com/. [Accessed July 1, 2013].

\section{AUTHOR BIOGRAPHIES}

CHARLES M. MACAL, PhD, PE, is the Director, Center for Complex Adaptive Agent Systems Simulation $\left(\mathrm{CAS}^{2}\right)$, Argonne National Laboratory. He is a member of the INFORMS-Simulation Society, Association for Computing Machinery, the Society for Computer Simulation International, and the System Dynamics Society. He is on the editorial boards of Transactions on Modeling and Computer Simulation, Simulation, and Complex Adaptive Systems Modeling. He has a Ph.D. in Industrial Engineering \& Management Sciences from Northwestern and a Master's Degree in Industrial Engineering from Purdue. Contact: macal@anl.gov. He is a registered professional engineer in the State of Illinois.

MICHAEL J. NORTH, MBA, PhD, is the Deputy Director, $\mathrm{CAS}^{2}$ at Argonne. He has over 15 years of experience developing advanced modeling and simulation applications for the federal government, international agencies, private industry, and academia. He has a Ph.D. in Computer Science from the Illinois Institute of Technology. Contact: north@anl.gov. 